\title{
High accuracy optical particle sizing in phase-Doppler anemometry
}

\author{
H-H Qiu $\dagger$, W Jia $\dagger$, C T Hsu $\dagger$ and M Sommerfeld $\ddagger$ \\ $\dagger$ Department of Mechanical Engineering, Hong Kong University of Science and Technology, \\ Kowloon, Hong Kong, People's Republic of China \\ \$Institut für Mechanische Verfahrenstechnik und Umweltschutztechnik, \\ Martin-Luther-Universität Halle-Wittenberg, Halle (Saale), Germany
}

Received 6 July 1999, in final form and accepted for publication 23 November 1999

\begin{abstract}
A novel structure for phase-Doppler anemometry (PDA) is proposed in order to eliminate the measurement-volume effect (MVE). This new PDA system consists of four receiving detectors in an optimized optical orientation. The method for the determination of the optimized optical orientation angle is introduced. In this method the vector sum of refractive and reflective rays is taken into consideration in describing a

dual-mechanism-scattering model caused by a nonuniformly illuminated PDA measurement volume. The constraint of the single-mechanism-scattering model in the conventional PDA is removed. As a result the error caused by MVE, which consists of a Gaussian beam defect and a slit effect, can be eliminated. Analytical and experimental studies were conducted to investigate the accuracy of particle sizing using this new system. Both the experimental results and the simulation based on the generalized Lorenz-Mie theory show that the new method can provide a high-accuracy PDA system free of the MVE.
\end{abstract}

Keywords: particle size, measurement-volume effect, phase-Doppler anemometry, optical particle sizing

\section{Introduction}

Phase-Doppler anemometry (PDA) for in situ measurements of spherical particles in multiphase flows is well developed nowadays. Many methods to enhance the performance of a PDA system, both in dynamic range and in accuracy, have been developed. Various developments have extended the application area of PDA, such as to measurements of mass flux and concentration, refractive index and temperature of particles in multiphase flows. Among these parameters, accurate measurement of the droplet size is of the utmost importance since the particle volume depends on the third power of the particle diameter. Because PDA is based on single-particle scattering theory, decreasing the measurement volume is often necessary to achieve highly accurate results under dense-particle conditions. Unfortunately a simple reduction in measurement volume can cause the phenomena to conflict with the assumption of uniform illumination. Consequently the result suffers from errors in sizing large particles owing to nonlinearity in the phase-diameter relationship because the beam intensity is practically nonuniform (Saffman 1986, Bachalo and Sankar 1988, Sankar et al 1992, Grehan et al 1991, Qiu and Sommerfeld 1992, Qiu and Hsu 1998a). This nonlinear effect is usually known as the measurement-volume effect (MVE) or trajectory ambiguity. Recent investigation with extended PDA has shown that the MVE also has a great effect on refractive-index-measurement results (Durst et al 1994). Numerical tools have been developed to simulate the Gaussian beam defect (see figure 1, where $P_{0}$ and $P_{1}$ denote reflection and refraction, respectively) by using the geometrical-optics theory (GOT) and the generalized Lorenz-Mie theory (GLMT) (Grehan et al 1991). To determine the effect of the slit image in the measurement volume, previous studies focused on the vignetting effect of the receiving optical aperture and slit (or pinhole) on the effective measurement volume. Experimental studies by Durst et al (1994) showed that the nonuniform-illumination effect due to the image boundary of the spatial slit filter in the receiving optics is even more critical than the Gaussianbeam defect (see figure 2). The Fourier-optics method recently developed by Qiu and Hsu (1996) has been used successfully to simulate the slit effect. Apparently the MVE consists of a Gaussian-beam defect and a slit effect that greatly hinder accuracy in particle sizing. Various solutions for minimizing trajectory ambiguity have been proposed for classical geometry (Qiu and Sommerfeld 1992) as well as more original designs such as planar geometry (Aizu et al 1993) and spatial-frequency geometry (Qiu and Hsu 1998b). A promising solution is dual-mode geometry, which integrates a standard PDA (SPDA) with a planar PDA (PPDA) (Tropea et al 1994). An important feature of PDA in a planar configuration is the temporal separation between the contributions of the two scattering mechanisms, reflection and refraction, in the light received by the photodetectors. By using the SPDA/PPDA phase ratio together with the burst-centring technique and the amplitude/size ratio, we may validate the scattering mechanism. This validation 


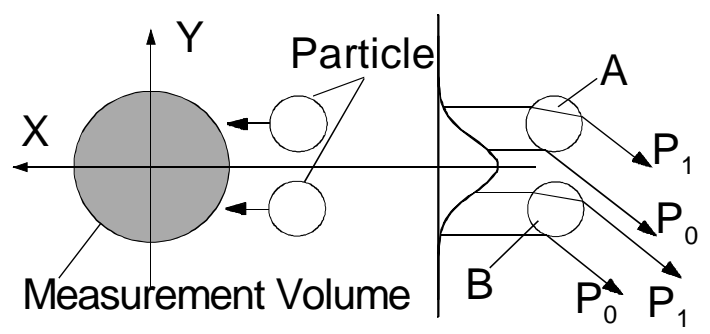

Figure 1. Scattering mechanisms caused by Gaussian beam illumination.

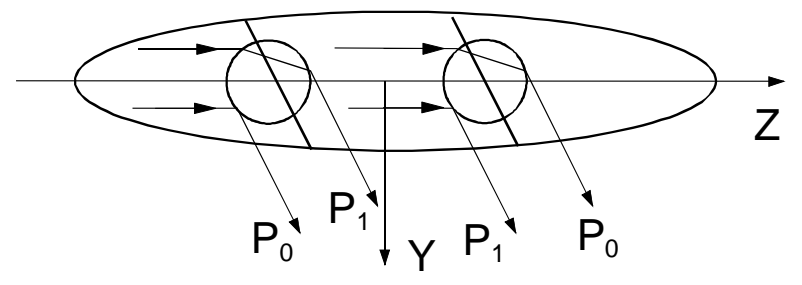

Figure 2. Scattering mechanisms caused by the slit effect.

implies the possibility of suppressing measurement errors due to mixing of the two modes of scattered light and therefore makes possible the performance of refractiveindex measurements by comparison of the reflective and the refractive phases. However, all the above methods for eliminating the MVE are based on the signal-validation scheme.

If the phase ratio or the amplitude/size ratio is not within the expected tolerance, the measured phase will be invalid. Because the effective measurement volume or crosssectional area is strongly dependent on the validation scheme, the methods reduce the measurement volume for those particles moving in a negative $Y$ position. The reduction in cross-sectional area in the $Y$ direction is particle-size dependent and it also results in the measurement volume being asymmetrical with regard to its intensity distribution. To correct this variation the measurement volume must be calibrated. Certainly this kind of correction is still feasible in a simple directional flow, such as in sprays. However, under complex two-phase-flow conditions, such as in swirling flows, due to arbitrary particle trajectories, the projection of the effective cross-sectional area for each particle size and direction of motion will be very complex. The result is that the calibration method could be very complicated. Furthermore, because PPDA has a relatively larger fluctuation in the size-phase relation than does SPDA when small particles are encountered, a validation method based only on the SPDA/PPDA phase ratio is not sufficient to eliminate the MVE completely, especially the slit effect that is also sensitive to small particles. The main reason for needing strong validation with the phase ratio and the amplitude/size ratio is only to identify the scattering mechanism. Because only one scattering mechanism was assumed in the conventional scattering model of PDA, any other scattering mechanisms are considered as error sources for size measurements. If a dual-mechanism-scattering model is developed by taking both the refraction and the reflection into consideration to determine particle size, no validation of the scattering mechanisms is necessary.
In this study a novel method based on a recently developed dual-mechanism-scattering model of Qiu and Hsu (1998a) was developed for calculating the size-phase correlation in PDA measurements. In this method two scattering phases measured by photodetectors are used to calculate the particle size. Because the fringe patterns from the refraction and the reflection are different in space and directions of motion, it has been found that there is an optimized optical orientation for which the particle diameter can be solved analytically. To demonstrate the capability of the newly developed method, the model was simulated numerically both by using GOT and by using the GLMT. Various optical parameters such as the measurement volume, the focal lengths of the sending and the receiving lenses, the size and the shape of the receiving aperture, the particle size and its trajectory and the phase-conversion factors are used to analyse the performance of this newly developed method.

\section{The analytical description}

The scheme of the newly proposed phase-Doppler system is shown in figure 3. The receiving optics consists of four detectors that are symmetrically orientated with elevation angles $\psi_{1}$ and $\psi_{2}$ for outer and inner pairs of detectors, respectively. The phase difference $\phi 14$ between the two outer detectors can be determined from the two Doppler signals. If we assume that the scattered light is dominated by refraction, according to GOT and taking the symmetrical elevation angle into consideration, the phase-size relation can be described as

$$
\phi 14_{1}=\phi 1_{1}-\phi 4_{1}=2 \phi 1_{1}=C 1_{1} D
$$

where $D$ is the particle diameter, $\phi 1_{1}$ and $\phi 4_{1}$ are the phases for detectors 1 and 4 , respectively, and $\phi 14_{1}$ is the phase difference between detectors 1 and 4 . The phase-conversion factor $C 1_{1}$ can be written as (Bauckhage 1988)

$$
\begin{gathered}
C 1_{1}=\frac{4 \pi}{\lambda}\left\{\left\{1+m^{2}-\sqrt{ } 2 m\left[1-\sin \left(\frac{\theta}{2}\right) \sin \psi_{1}\right.\right.\right. \\
\left.\left.\left.+\cos \left(\frac{\theta}{2}\right) \cos \psi_{1} \cos \varphi\right]^{1 / 2}\right\}^{1 / 2}\right\} \\
-\left\{\left\{1+m^{2}-\sqrt{ } 2 m\left[1+\sin \left(\frac{\theta}{2}\right) \sin \psi_{1}\right.\right.\right. \\
\left.\left.\left.+\cos \left(\frac{\theta}{2}\right) \cos \psi_{1} \cos \varphi\right]^{1 / 2}\right\}^{1 / 2}\right\}
\end{gathered}
$$

where $m$ is the particle refractive index and angles $\theta, \varphi$ and $\psi_{1}$ are defined in figure 3 .

Equation (1) is true only when the scattering is dominated by refraction. If the scattered light is dominated by reflection, the phase-conversion factor must be changed to

$$
\begin{aligned}
C 1_{0} & =\frac{2 \pi \sqrt{ } 2}{\lambda}\left\{\left[1+\sin \left(\frac{\theta}{2}\right) \sin \psi_{1}\right.\right. \\
& \left.-\cos \left(\frac{\theta}{2}\right) \cos \psi_{1} \cos \varphi\right]^{1 / 2}-\left[1-\sin \left(\frac{\theta}{2}\right) \sin \psi_{1}\right. \\
& \left.\left.-\cos \left(\frac{\theta}{2}\right) \cos \psi_{1} \cos \varphi\right]^{1 / 2}\right\} .
\end{aligned}
$$




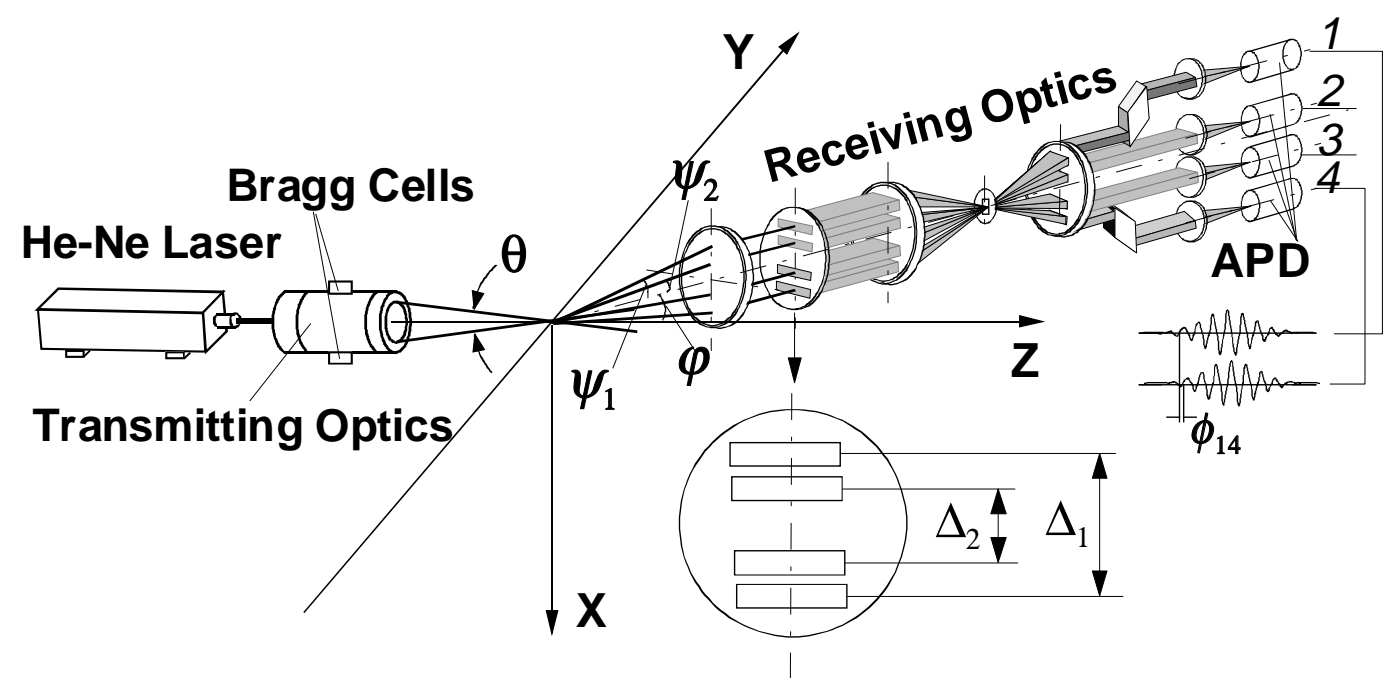

Figure 3. The optical layout of four-detector phase-Doppler anemometry. APD, avalanche photodiode.

The phase-size relation for the detector pairs $\phi 14_{0}$ can also be described as

$$
\phi 14_{0}=2 \pi-\left(\phi 1_{0}-\phi 4_{0}\right)=2 \pi-2 \phi 1_{0}=2 \pi-C 1_{0} D .
$$

The difference between the forms of equations (1) and (4) shows that the reflective fringe pattern moves in the direction opposite to that of the refractive pattern. Hence, if one of the scattering mechanisms can be measured separately, either equation (1) or equation (4) can be used to calculate the particle diameter. Unfortunately the problem is not so simple in practical implementation. For the problem to be better understood, a vector-sum model of the dual scattering mechanisms was developed, as shown in figure 4 , in which several possibilities for combining the vector sum for refraction and reflection are described. In figure 4 it is clear that both refraction and reflection, which coexist in most cases, contribute to the measured phase. Therefore the problem is not one of domination by either reflection or refraction but rather a combination of both. According to the vector analysis in figure 4 and the phase-size relations of single-mechanism scattering in equations (1) and (4), the functions describing the phase difference between detectors 1 and $4, \phi 14$, from dual-scattering mechanisms are obtained in the following form:

$$
\begin{gathered}
\phi 14=2 \phi 1=2 \arctan \left(\frac{I 1_{1} \sin \phi 1_{1}-I 1_{0} \sin \phi 1_{0}}{I 1_{1} \cos \phi 1_{1}-I 1_{0} \cos \phi 1_{0}}\right) \\
=2 \arctan \left(\frac{I 1_{1} \sin \left(\frac{C 1_{1} D}{2}\right)-I 1_{0} \sin \left(\frac{C 1_{0} D}{2}\right)}{I 1_{1} \cos \left(\frac{C 1_{1} D}{2}\right)+I 1_{0} \cos \left(\frac{C 1_{0} D}{2}\right)}\right)
\end{gathered}
$$

where $I 1_{1}$ and $I 1_{0}$ are the received intensities of refracted and reflected rays, respectively.

If $I 1_{1}$ and $I 1_{0}$ can be measured experimentally or determined analytically, we can uniquely quantify the phasesize relation by using equation (5). Unfortunately no method of separately measuring the intensity of each ray is available yet, so it is difficult for us to calculate the phase-size relation directly by using equation (5). To solve this problem, let us introduce an additional pair of detectors, namely detectors
2 and 3 shown in figure 3 . The phase difference, $\phi 23$, can be written similarly to that for detectors 1 and 4 by substituting $I 1_{1}, I 1_{0}, C 1_{1}$ and $C 1_{0}$ by $I 2_{1}, I 2_{0}, C 2_{1}$ and $C 2_{0}$ in equation (5), respectively.

In a practical PDA system the elevation angle of each pair of detectors is quite small and the sensitivities of avalanche photodiodes are adjusted to achieve almost identical amplitude in each output signal. Hence the scattering intensities can be assumed the same for each detector. In this case $I 1_{1} \approx I 2_{1}=I_{1}$ and $I 1_{0} \approx I 2_{0}=I_{0}$ can be assumed, which has also been proved by GLMT simulations. Furthermore, if the optimized off-axis angle is selected to have equal phase-conversion factors for refractive and reflective rays (Qiu and Hsu 1998b), i.e. $C 1_{1}=C 1_{0}=$ $C_{1}$ and $C 2_{1}=C 2_{0}=C_{2}$, equation (5) can be rewritten as

$$
\begin{aligned}
& \phi 14=2 \arctan \left[\frac{I_{1}-I_{0}}{I_{1}+I_{0}} \tan \left(\frac{C_{1} D}{2}\right)\right] \\
& \phi 23=2 \arctan \left[\frac{I_{1}-I_{0}}{I_{1}+I_{0}} \tan \left(\frac{C_{2} D}{2}\right)\right] .
\end{aligned}
$$

Hence the relationship between the measured phases, $\phi 14$ and $\phi 23$, and the particle diameter $D$ can be written as

$$
\frac{\tan \left(\frac{\phi 14}{2}\right)}{\tan \left(\frac{\phi 23}{2}\right)}=\frac{\tan \left(\frac{C_{1} D}{2}\right)}{\tan \left(\frac{C_{2} D}{2}\right)} .
$$

Because only the particle diameter $D$ in equation (7) is unknown, it can be solved. A further optimization was found by the choice of $C_{1}=2 C_{2}$. As a result the particle diameter can be calculated as follows:

$$
D=\frac{4}{C_{1}} \arctan \left[\left(1-\frac{2 \tan \left(\frac{\phi 23}{2}\right)}{\tan \left(\frac{\phi 14}{2}\right)}\right)^{1 / 2}\right]
$$

where $\phi 14$ and $\phi 23$ can be determined experimentally. 


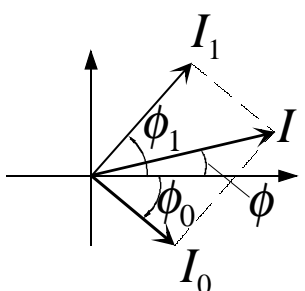

(a)

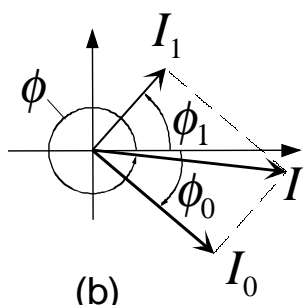

(b)

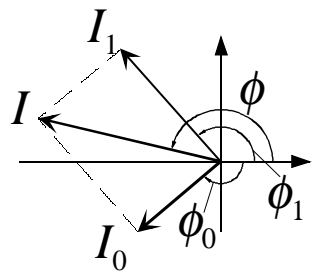

(c)

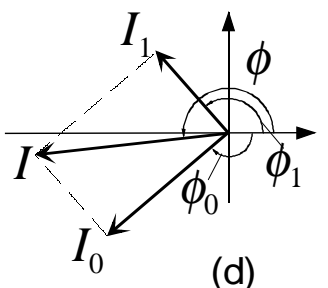

(d)

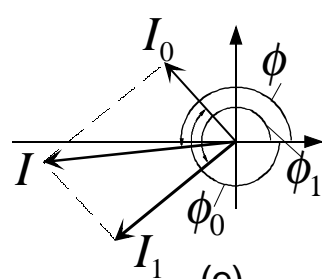

(e)

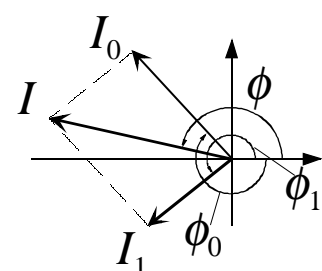

(f)

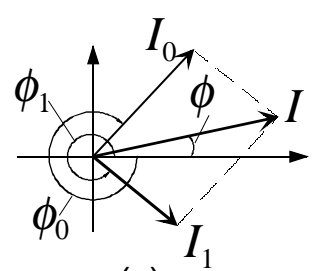

(g)

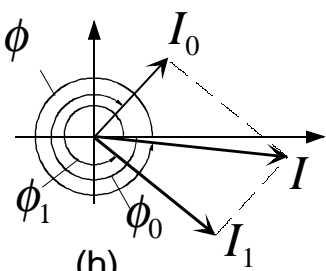

(h)

Figure 4. Vector analysis of the superposition of refracted and reflected rays.

The optimized off-axis angle $\varphi$ for the receiving detectors can be written as

$$
\varphi=\left\{\begin{array}{cc}
\arccos \left[\left(\frac{1}{4 m^{2}}\left[1+4 m^{2}+\left(1+8 m^{2}\right)^{1 / 2}\right]-1\right)\right] \\
\text { for } m>1 & \text { for } m<1 \\
\arccos \left(2 m^{2}-1\right) &
\end{array}\right.
$$

(see the appendix). Hence, the optimized off-axis angle is a function of the relative refractive index of the media only. It is important to point out that, because the optimized offaxis angle is independent of the optical geometry, the angle determined by equation (9) is also the optimized angle for the pairs of detectors 2 and 3 .

\section{GLMT simulations and discussion}

To evaluate the performance of equation (8) that is based on the dual-scattering-mechanism model, the GLMT was adopted to simulate the MVE compared with that based on the conventional single-scattering-mechanism assumption. The optimized off-axis angles satisfying equation (9), (2) and (3) were calculated by using an off-axis angle range from $20^{\circ}$ to $80^{\circ}$ (see figure 5). The results of calculation show that a $46.25^{\circ}$ satisfies the requirement for both pairs of detectors 1 and 4 and 2 and 3 . The results of numerical calculation for a relative refractive-index range from 0.75 to 2.0 are shown in figure 6 , which can be used as a guideline for the design of the optical orientation angle in practice if this newly developed method is implemented.

Simulations of the MVE were carried out for the system geometry shown in figure 3 with the parameters given in table 1 . For the conventional method only the outer pairs of detectors was used in the simulation. Two major directions of particle motion, namely $Y$ and $X$, were considered. They

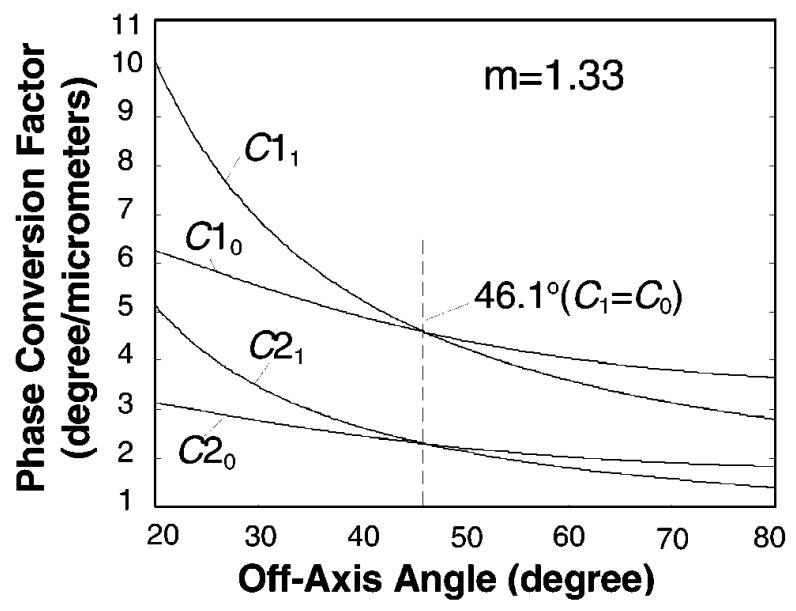

Figure 5. Determination of the $C_{1}=C_{0}$ point using equations (2) and (3).

are the ones most commonly encountered in practical cases. The results of simulation are shown in figures 7-13. As shown in figure 7 a $60 \mu \mathrm{m}$ water droplet passing through the measurement volume parallel to the $Y$ axis at $z=0 \mu \mathrm{m}$ will yield a phase error of $27 \%$ owing to the change of the scattering mechanism from reflection to refraction. If the phase is determined by the conventional method, i.e. directly calculated by use of equation (1), a large measurement error may occur. Only a very small fluctuation $(<1 \%)$ in the phase determination using equation (8) and hence in the MVE, can be neglected. Figures 8 and 9 show $49 \%$ and $45 \%$ errors obtained by using the conventional method for 70 and $80 \mu \mathrm{m}$ droplets, respectively. However, the error is less than $1 \%$ using the new approach. A very large phase transition was observed in figure 10 for a $90 \mu \mathrm{m}$ droplet passing through the measurement volume. Although in this case the maximum phase error almost reaches $90 \%$ with 


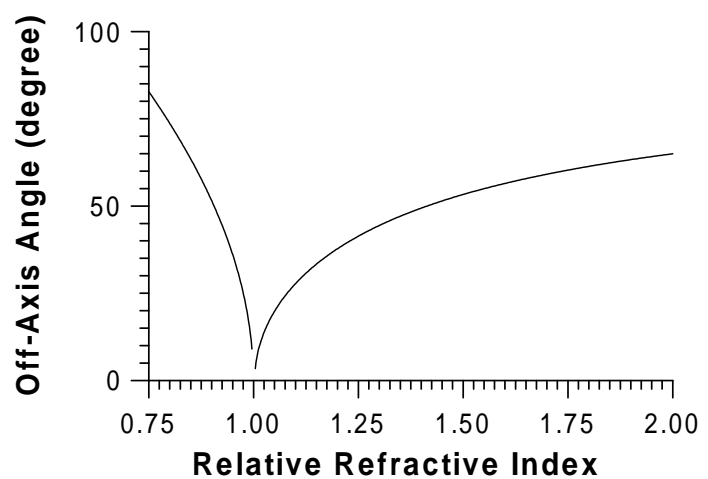

Figure 6. The dependence of the MVE-free line on the off-axis angle and the relative refractive index of the media.

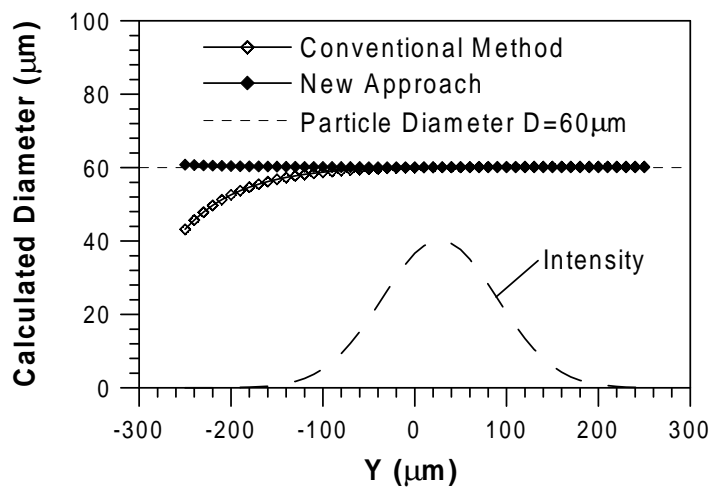

Figure 7. A comparison of size-estimation results between the conventional method and the new approach $(X=Z=0 \mu \mathrm{m}$ and $\left.r_{0}=125 \mu \mathrm{m}\right)$.

Table 1. Optical parameters.

\begin{tabular}{ll}
\hline Parameters & Value \\
\hline Wavelength, $\lambda(\mu \mathrm{m})$ & 0.5145 \\
Radius of beam waist, $r_{0}(\mu \mathrm{m})$ & 125 \\
Half transmitting angle (degrees) & 1.503 \\
Receiving elevation angle 1 (degrees) & 4.686 \\
Receiving elevation angle 2 (degrees) & 2.343 \\
Off-axis angle (degrees) & 46.25 \\
Refractive index & 1.33 \\
\hline
\end{tabular}

the conventional method, the measurement error with the new approach is still only about $3 \%$. As a result, although the scattering's alternating between reflection and refraction yields a large phase change in the conventional method, the calculated phase when the new method is used is almost constant and is proportional to the particle diameter. Note that the measured phases in figures 7-10 at the centre of the burst (the maximum Gaussian-envelope position) are correct both for the conventional and for the new method. Hence a burst-centring signal-processing technique can eliminate errors from the measurements when particles pass through the measurement volume in the $Y$ direction, as has been reported by Qiu et al (1994).

The MVE for droplets moving in the $X$ direction is shown in figures 11-14 for droplet diameters of 15, 30, 50 and $70 \mu \mathrm{m}$, respectively. In figure 11 , the diameters measured with the conventional and the newly developed

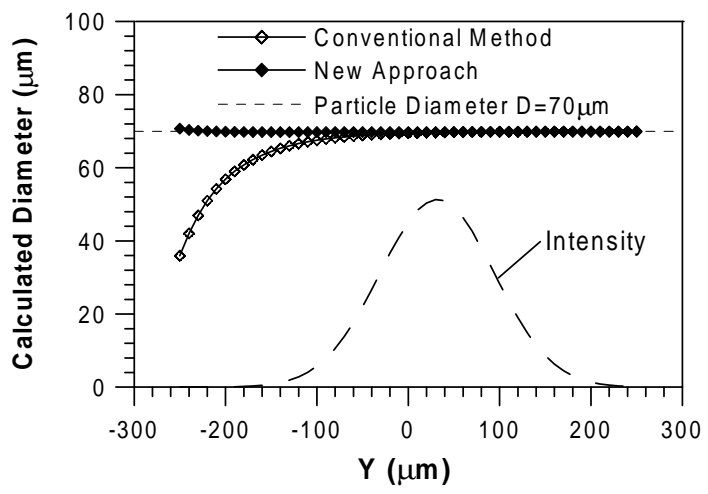

Figure 8. A comparison of size-estimation results between the conventional method and the new approach $(X=Z=0 \mu \mathrm{m}$ and $\left.r_{0}=125 \mu \mathrm{m}\right)$

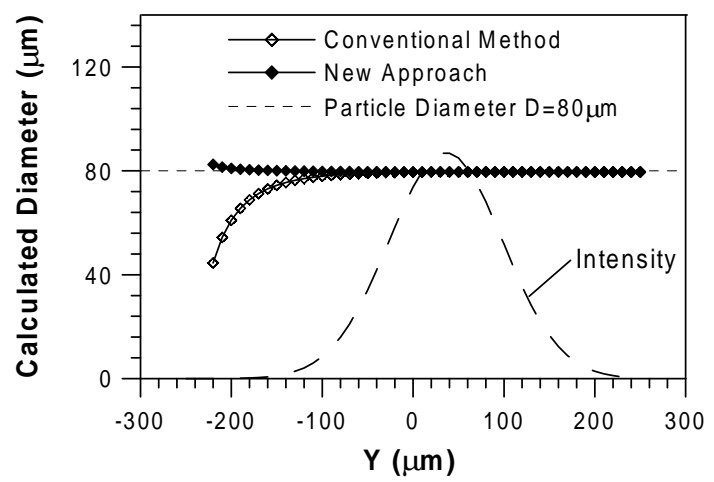

Figure 9. A comparison of size-estimation results between the conventional method and the new approach $(X=Z=0 \mu \mathrm{m}$ and $\left.r_{0}=125 \mu \mathrm{m}\right)$.

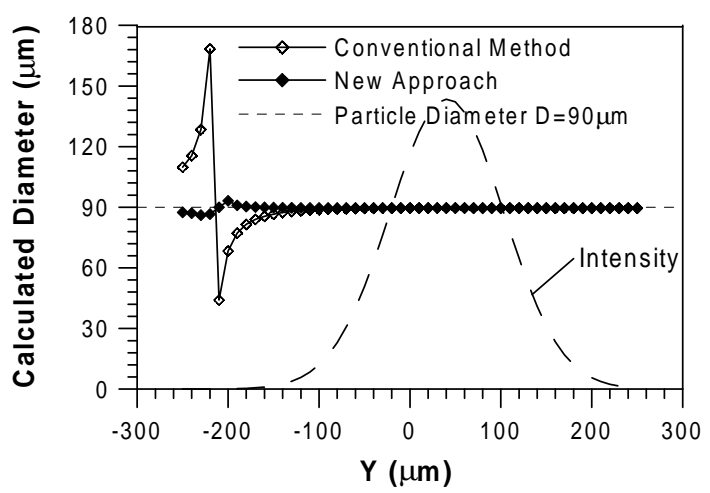

Figure 10. A comparison of size-estimation results between the conventional method and the new approach $(X=Z=0 \mu \mathrm{m}$ and $\left.r_{0}=125 \mu \mathrm{m}\right)$

methods are almost identical to the ideal diameter calculated by use of equation (1). This is because, for a given droplet diameter smaller than one fifth of the measurement-volume diameter, the MVE can normally be neglected. If the droplet diameter is larger than one fifth of the diameter of the measurement volume, the MVE becomes significant, as shown in figures 12-14. In figures 12-14 droplets with diameters of 60,70 and $80 \mu \mathrm{m}$, respectively, passing trough the measurement volume in the $X$ direction $(Y=-220$, $Z=0$ ), were simulated. The conventional method gives 


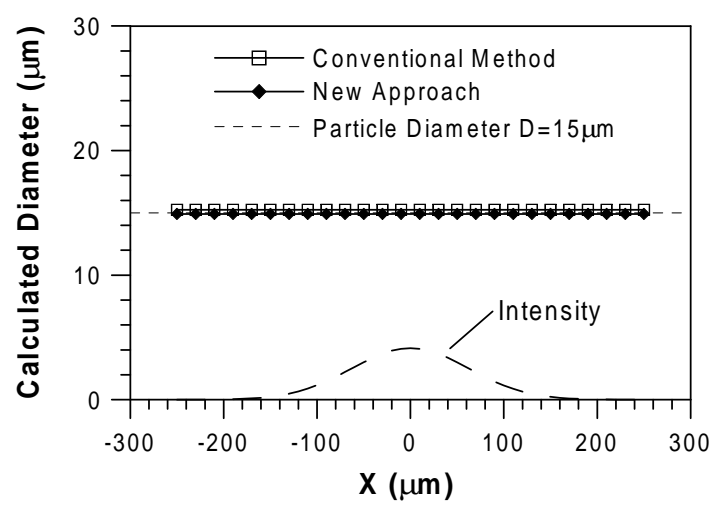

Figure 11. A comparison of size-estimation results between the conventional method and the new approach $(Y=-220 \mu \mathrm{m}$, $Z=0 \mu \mathrm{m}$ and $r_{0}=125 \mu \mathrm{m}$ ).

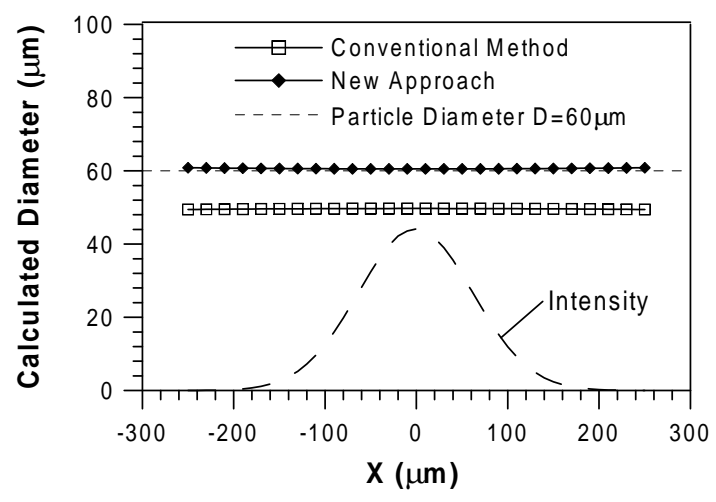

Figure 12. A comparison of size-estimation results between the conventional method and the new approach $(Y=-220 \mu \mathrm{m}$, $Z=0 \mu \mathrm{m}$ and $\left.r_{0}=125 \mu \mathrm{m}\right)$.

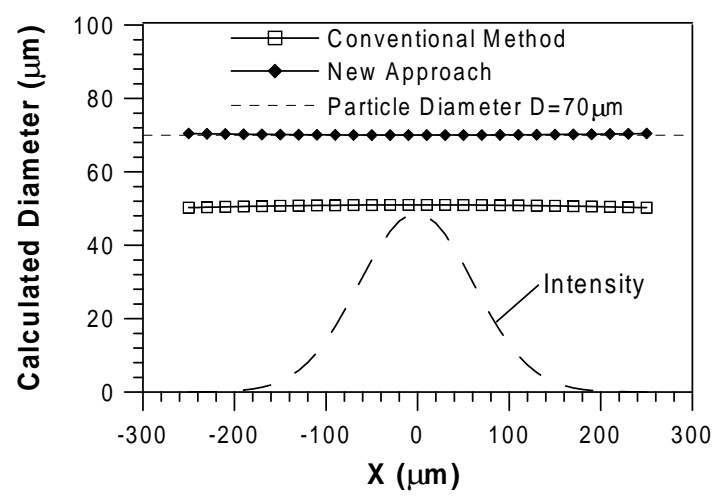

Figure 13. A comparison of size-estimation results between the conventional method and the new approach $(Y=-220 \mu \mathrm{m}$, $Z=0 \mu \mathrm{m}$ and $\left.r_{0}=125 \mu \mathrm{m}\right)$.

$16 \%, 29 \%$ and $50 \%$ diameter-measurement errors constantly throughout the droplet trajectory in the measurement volume. Therefore, even when the peak-detector (burst-centring) technique is used, measurement errors cannot be eliminated. The newly developed method provides measurement errors of less than $2 \%$. The MVEs for 60 and $80 \mu \mathrm{m}$ droplets moving along $Z$ direction are shown in figures 15 and 16 , respectively. Because the illuminance along the $Z$ direction is almost constant, the dominance of scattering mechanisms

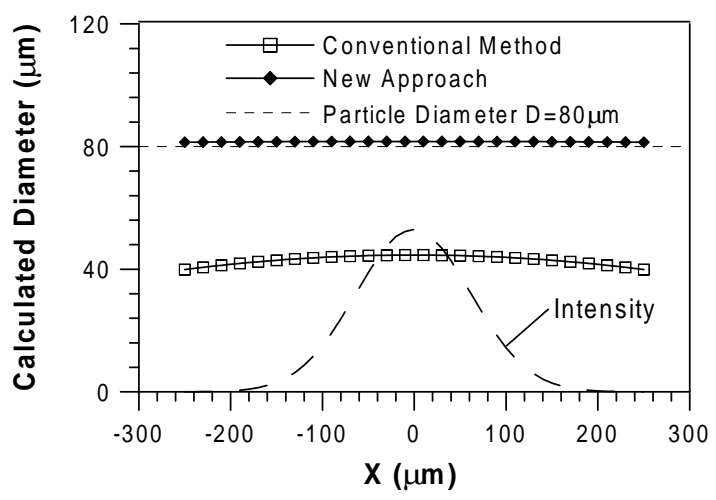

Figure 14. A comparison of size-estimation results between the conventional method and the new approach $(Y=-220 \mu \mathrm{m}$, $Z=0 \mu \mathrm{m}$ and $\left.r_{0}=125 \mu \mathrm{m}\right)$.

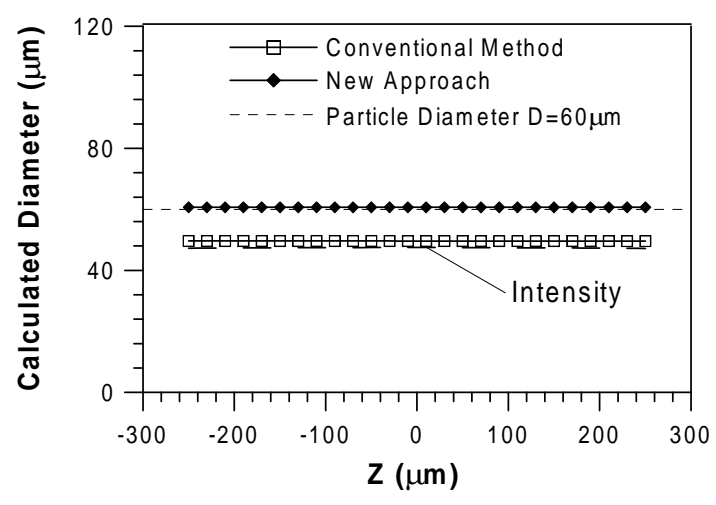

Figure 15. A comparison of size-estimation results between the conventional method and the new approach $(Y=-220 \mu \mathrm{m}$, $X=0 \mu \mathrm{m}$ and $\left.r_{0}=125 \mu \mathrm{m}\right)$.

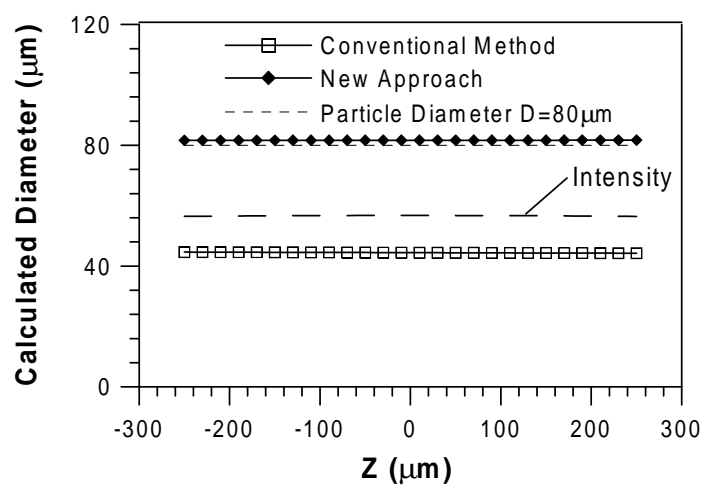

Figure 16. A comparison of size-estimation results between the conventional method and the new approach $(Y=-220 \mu \mathrm{m}$, $X=0 \mu \mathrm{m}$ and $\left.r_{0}=125 \mu \mathrm{m}\right)$.

is not alternating if a particle is moving along the $Z$ direction. Hence, it is expected that the behaviours of MVEs are almost identical along the $Z$ direction, i.e. the behaviour of the MVE for a particle moving in the $Z=0$ plane (the $X-Y$ plane) can be used to describe that in any $Z \neq 0$ plane. Figures 15 and 16 further provide the proof of this estimation, in that the MVE is almost constant in the $Z$ direction and the new approach provides a significant improvement over the conventional method. 


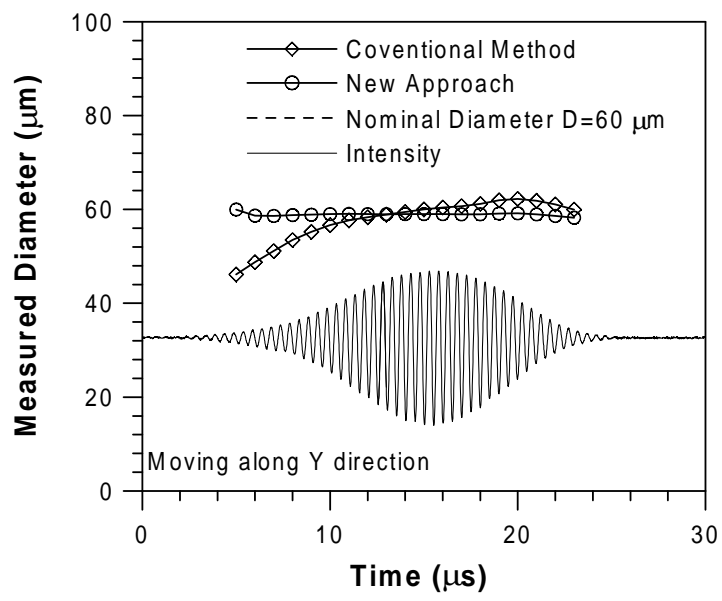

Figure 17. A comparison of measurement results between the conventional method and the new approach $(X=Z=0 \mu \mathrm{m}$ and $\left.r_{0}=125 \mu \mathrm{m}\right)$.

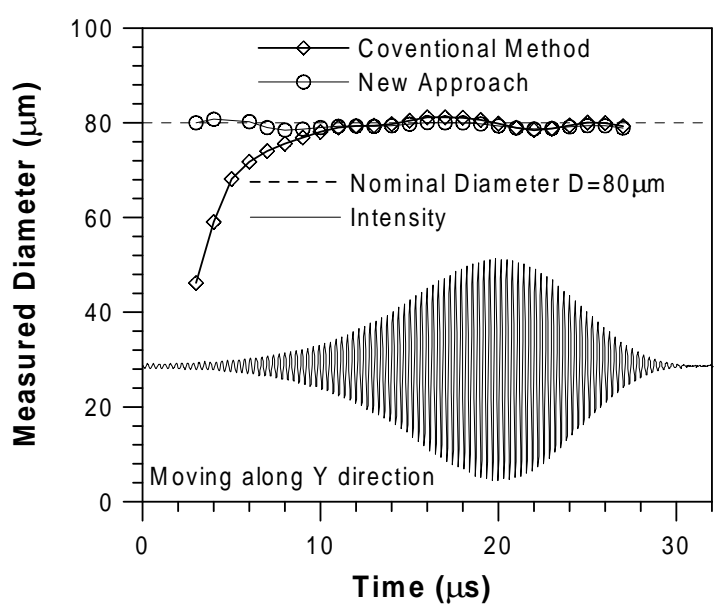

Figure 18. A comparison of measurement results between the conventional method and the new approach $(Y=Z=0 \mu \mathrm{m}$ and $\left.r_{0}=125 \mu \mathrm{m}\right)$.

\section{Validation experiments and discussion}

To further validate the proposed method, a PDA system based on figure 3 was developed. Experiments were conducted by traversing monodispersed water droplets, which were generated by a TSI monodispersed-droplet generator, through the measurement volume in the $X$ and $Y$ directions. The parameters of the receiving units, shown in table 1, make the results comparable with the GLMT simulations. Tow sets of Dantect/Invent PDE systems and a four-channel transient recorder (LeCroy 9153MT) were used to perform signal conditioning and data acquisition. A recently developed SNR burst detector was used to trigger the system. During the measurements, whole Doppler bursts from the four channels were stored in the transient recorder for post-processing. The recorded data were then transferred to the computer and evaluated by a cross-spectral-density (CSD) and five-point-fitting method. The calculated phase pairs $\phi 14$ and $\phi 23$ were substituted into equation (8) for calculating the droplet diameter. Similarly to the GLMT simulation, two major directions of particle motion were

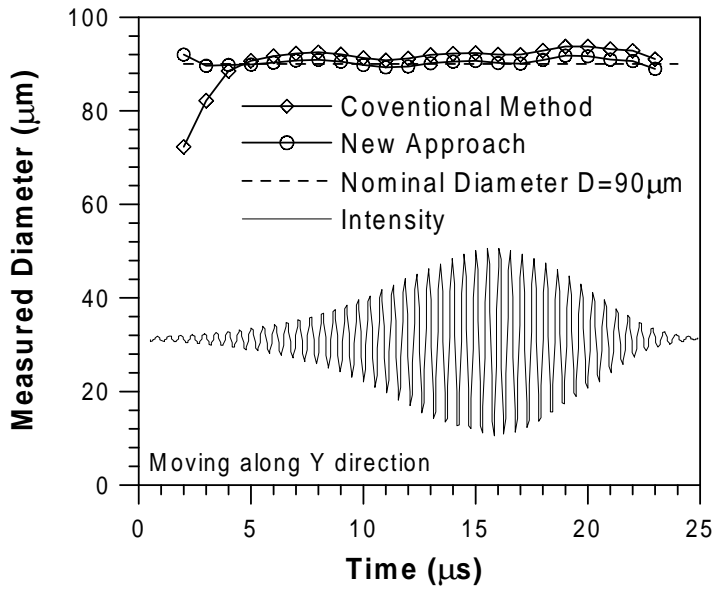

Figure 19. A comparison of measurement results between the conventional method and the new approach $(Y=Z=0 \mu \mathrm{m}$ and $\left.r_{0}=125 \mu \mathrm{m}\right)$.

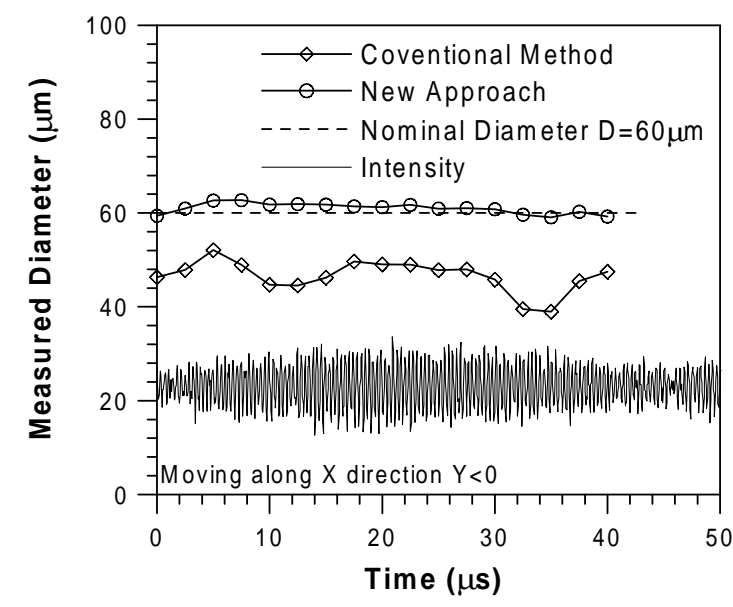

Figure 20. A comparison of measurement results between the conventional method and the new approach $(Y<0, Z=0 \mu \mathrm{m}$ and $\left.r_{0}=125 \mu \mathrm{m}\right)$.

validated. It was difficult to exactly measure particle positions. Therefore, the scattering intensities were used to identify $Y>0$ and $Y<0$ positions. In the experiments, if the scattering intensity from the particle reached the maximum in comparison with that of other trajectories, from the geometrical-optical theory we can assume that the particle was traversing the measurement volume through a positive $Y$ position. If the particle passed the measurement volume through a negative $Y$ position, the scattering intensity would be reduced. Therefore, by selecting the scattering intensity reduced to a certain threshold while the nozzle was traversing towards the negative $Y$ direction, the $Y<0$ position can be identified. Figures 17-19 show the measurement results for droplets of diameters 60,80 and $90 \mu \mathrm{m}$ passing through the measurement volume parallel to the $Y$ axis at $z=x=0 \mu \mathrm{m}$. The experimental results show that, using the conventional method, large measurement errors may occur if the triggering signals are inappropriately selected. In particular, if the trigger is starting from the beginning of the burst, the maximum measurement errors under current testing conditions may reach $24 \%, 43 \%$ and $10 \%$ for 60 , 


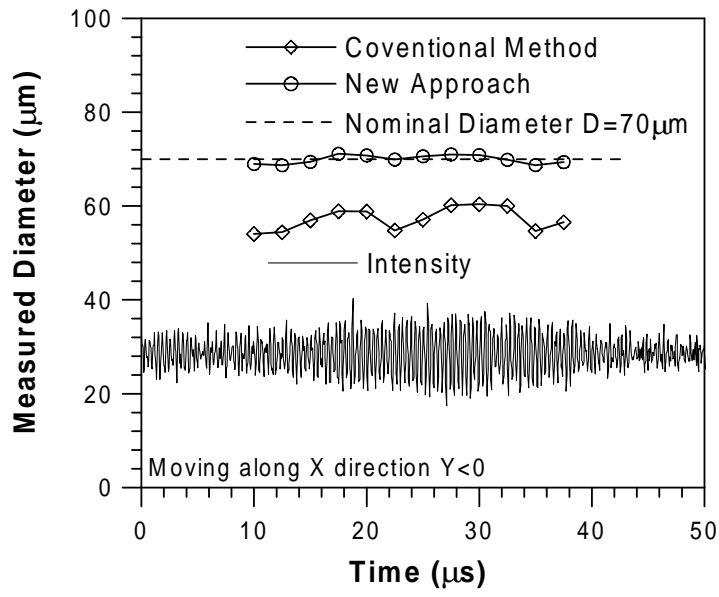

Figure 21. A comparison of measurement results between the conventional method and the new approach $(Y<0 \mu \mathrm{m}$, $Z=0 \mu \mathrm{m}$ and $\left.r_{0}=125 \mu \mathrm{m}\right)$.

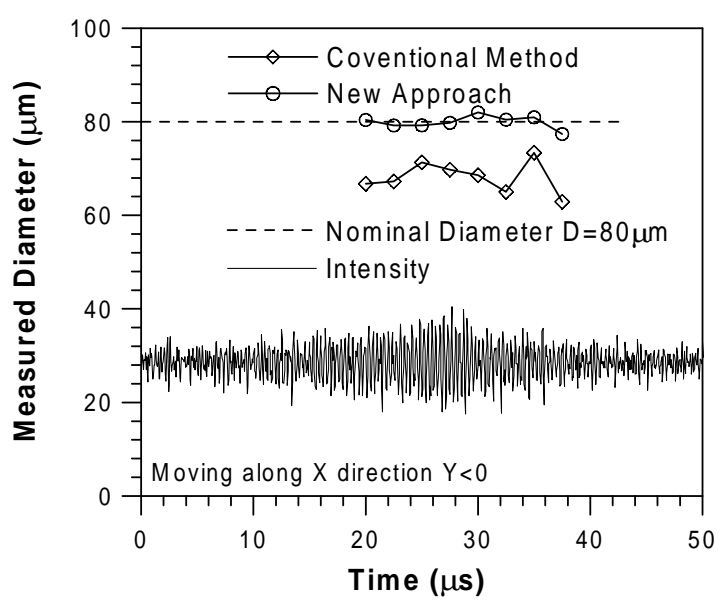

Figure 22. A comparison of measurement results between the conventional method and the new approach $(Y<0 \mu \mathrm{m}$, $Z=0 \mu \mathrm{m}$ and $\left.r_{0}=125 \mu \mathrm{m}\right)$.

80 and $90 \mu \mathrm{m}$ droplets, respectively. Similarly to the GLMT simulation results, the new method demonstrates the capability for eliminating the MEV and provides maximum measurement errors of less than 3\%. Figures 20-22 give the experimental results for droplets of diameters 60, 70 and $80 \mu \mathrm{m}$ passing through the measurement volume parallel to the $X$ direction with $Z=0$ and $Y<0$. As we mentioned previously, this direction is the most critical one since the peak detector (burst centring) cannot help one to eliminate the MVE in this direction of particle motion. The maximum measurement errors are about $20-30 \%$ in all cases. However, the new method once again shows its capability for eliminating the MVE by providing maximum measurement errors of less than $5 \%$ in all cases. Although the maximum measurement error is slightly larger than that in the $Y$ direction, we believe that was caused by the poor SNR values of the signals.

\section{Conclusion}

A novel method for PDA measurement free of the MVE was developed. This new method can effectively eliminate the MVE including Gaussian beam defects and slit effects, by using a four-detector PDA system that is very easy to implement in two-phase-flow measurements. Because no strong validation scheme is necessary other than measurement of the SNR, the measurement volume can be accurately determined by conventional methods and hence we have high accuracy in the particle-mass-flux and concentration measurements. This new system has the same transmitting optics as a conventional three-detector PDA system but with optimized orientation of the receiving units. The method for determining the optimized off-axis angle was introduced and its equation was derived. The performance of this new method was simulated by using the GLMT and validated by experiments. We described the newly developed model in equation (8) for the phase-size correlation by using a geometrical optics method, which is especially suitable for sizing large particles. Therefore, it is expected that this new method will provide a highly accurate PDA system free of Gaussian beam defects and slit effects for multiphase-flow measurements.

\section{Acknowledgments}

This research was supported by the Hong Kong Government and Hong Kong University of Science and Technology under RGC/EGR (Research Grants Council/Earmarked Grant for Research) grant HKUST 6108/97E.

\section{Appendix}

To derive the optimized off-axis angle $\varphi$ for the pair of detectors 1 and 4 it is simply necessary to rewrite equation (2) as

$$
\begin{aligned}
C 1_{1} & =\frac{4 \pi}{\lambda}\left\{\left[1+m^{2}-\sqrt{ } 2 m\left[1+\cos \left(\frac{\theta}{2}\right) \cos \psi_{1} \cos \varphi\right]^{1 / 2}\right.\right. \\
& \left.\times\left(1-\frac{\sin \left(\frac{\theta}{2}\right) \sin \psi_{1}}{1+\cos \left(\frac{\theta}{2}\right) \cos \psi_{1} \cos \psi}\right)^{1 / 2}\right]^{1 / 2} \\
& -\left[1+m^{2}-\sqrt{ } 2 m\left[1+\cos \left(\frac{\theta}{2}\right) \cos \psi_{1} \cos \varphi\right]^{1 / 2}\right. \\
& \left.\left.\times\left(1+\frac{\sin \left(\frac{\theta}{2}\right) \sin \psi_{1}}{1+\cos \left(\frac{\theta}{2}\right) \cos \psi_{1} \cos \psi}\right)^{1 / 2}\right]^{1 / 2}\right\} .
\end{aligned}
$$

In practical PDA systems the transmitting and receiving elevation angles are very small; hence, by applying the approximations

$$
\begin{aligned}
\frac{\sin \left(\frac{\theta}{2}\right) \sin \psi_{1}}{1+\cos \left(\frac{\theta}{2}\right) \cos \psi_{1} \cos \varphi} \ll 1 & \ll 1 \pm \frac{a}{2} \quad|a| \ll 1 .
\end{aligned}
$$

Equation (A1) can be rewritten as

$$
\begin{aligned}
C 1_{1} & =\frac{4 \pi}{\lambda}\left[\left(1+m^{2}-\sqrt{ } 2 m\left[1+\cos \left(\frac{\theta}{2}\right) \cos \psi_{1} \cos \varphi\right]^{1 / 2}\right.\right. \\
& \left.+\frac{m}{\sqrt{ } 2} \sin \left(\frac{\theta}{2}\right) \sin \psi \frac{1}{\left[1+\cos \left(\frac{\theta}{2}\right) \cos \psi_{1} \cos \varphi\right]^{1 / 2}}\right)^{1 / 2} \\
& -\left(1+m^{2}-\sqrt{ } 2 m\left[1+\cos \left(\frac{\theta}{2}\right) \cos \psi_{1} \cos \varphi\right]^{1 / 2}\right.
\end{aligned}
$$


H-H Qiu et al

$$
\left.\left.-\frac{m}{\sqrt{ } 2} \sin \left(\frac{\theta}{2}\right) \sin \psi_{1} \frac{1}{\left[1+\cos \left(\frac{\theta}{2}\right) \cos \psi_{1} \cos \varphi\right]^{1 / 2}}\right)^{1 / 2}\right] .
$$

After further reformulating equation (A3), we have

$$
\begin{aligned}
C 1_{1} & =\frac{4 \pi}{\lambda}\left[\left\{1+m^{2}-\sqrt{ } m\left[1+\cos \left(\frac{\theta}{2}\right) \cos \psi_{1} \cos \varphi\right]^{1 / 2}\right\}^{1 / 2}\right. \\
& \times\left(1+\frac{\frac{m}{\sqrt{ } 2} \sin \left(\frac{\theta}{2}\right) \sin \psi_{1} \frac{1}{\left[1+\cos \left(\frac{\theta}{2}\right) \cos \psi_{1} \cos \varphi\right]^{1 / 2}}}{1+m^{2}-\sqrt{ } 2 m\left[1+\cos \left(\frac{\theta}{2}\right) \cos \psi_{1} \cos \varphi\right]^{1 / 2}}\right)^{1 / 2} \\
& \left.-\left(1-\frac{\frac{m}{\sqrt{ } 2} \sin \left(\frac{\theta}{2}\right) \sin \psi_{1} \frac{1}{\left.1+\cos \left(\frac{\theta}{2}\right) \cos \psi_{1} \cos \varphi\right]^{1 / 2}}}{1+m^{2}-\sqrt{ } 2 m\left[1+\cos \left(\frac{\theta}{2}\right) \cos \psi_{1} \cos \varphi\right]^{1 / 2}}\right)^{1 / 2}\right] .
\end{aligned}
$$

Once again, by applying a similar approximation to that used for equation (A2) to equation (A4), we have

$$
\begin{aligned}
C 1_{1} & =\frac{4 \pi}{\sqrt{ } 2 \lambda}\left[m \sin \left(\frac{\theta}{2}\right) \sin \psi_{1}\right] \\
& \times \llbracket\left\{1+m^{2}-\sqrt{ } 2 m\left[1+\cos \left(\frac{\theta}{2}\right) \cos \psi_{1} \cos \varphi\right]^{1 / 2}\right\}^{1 / 2} \\
& \left.\times\left[1+\cos \left(\frac{\theta}{2}\right) \cos \psi_{1} \cos \varphi\right]^{1 / 2}\right]^{-1} .
\end{aligned}
$$

Similarly, equation (3) can be reduced to

$$
\begin{aligned}
C 1_{0} & =\frac{2 \pi \sqrt{ } 2}{\lambda}\left\{\left[1-\cos \left(\frac{\theta}{2}\right) \cos \psi_{1} \cos \varphi\right.\right. \\
& \left.+\sin \left(\frac{\theta}{2}\right) \sin \psi_{1}\right]^{1 / 2}-\left[1-\cos \left(\frac{\theta}{2}\right) \cos \psi_{1} \cos \varphi\right. \\
& \left.\left.-\sin \left(\frac{\theta}{2}\right) \sin \psi_{1}\right]^{1 / 2}\right\} \\
= & \frac{2 \pi \sqrt{ } 2}{\lambda} \frac{\sin \left(\frac{\theta}{2}\right) \sin \psi_{1}}{\left[1-\cos \left(\frac{\theta}{2}\right) \cos \psi_{1} \cos \varphi\right]^{1 / 2}} .
\end{aligned}
$$

By equating equations (A5) and (A6), we have

$$
\begin{aligned}
m \llbracket & \left\{1+m^{2}-\sqrt{ } 2 m\left[1+\cos \left(\frac{\theta}{2}\right) \cos \psi_{1} \cos \varphi\right]^{1 / 2}\right\}^{1 / 2} \\
& \left.\times\left[1+\cos \left(\frac{\theta}{2}\right) \cos \psi_{1} \cos \varphi\right]^{1 / 2}\right]^{-1} \\
= & \frac{1}{\left[1-\cos \left(\frac{\theta}{2}\right) \cos \psi_{1} \cos \varphi\right]^{1 / 2}} .
\end{aligned}
$$

Let

$$
\eta=1+\cos \left(\frac{\theta}{2}\right) \cos \psi_{1} \cos \varphi
$$

Equation (A7) can be rewritten as

$$
2 m^{2} \eta^{3}-\left(4 m^{4}+4 m^{2}+1\right) \eta^{2}+4 m^{2}\left(2 m^{2}+1\right) \eta-4 m^{4}=0 .
$$

The solutions of equation (A9) are

$$
\eta=\left\{\begin{array}{l}
2 m^{2} \\
1+\frac{1}{4 m^{2}}\left[1+\left(1+8 m^{2}\right)^{1 / 2}\right] \\
1+\frac{1}{4 m^{2}}\left[1-\left(1+8 m^{2}\right)^{1 / 2}\right] .
\end{array}\right.
$$

From equation (A8) the solutions equation (A10) must also satisfy the inequality, i.e. $1<\eta<2$ for an off-axis angle $0<\varphi<90^{\circ}$. Hence, only the solutions $\eta=2 \mathrm{~m}^{2}$ and

$$
\eta=1+\frac{1}{4 m^{2}}\left[1+\left(1+8 m^{2}\right)^{1 / 2}\right]
$$

are meaningful to the conditions $m<1$ and $m>1$, respectively. By substituting the solution (21) into equation (A8), the optimized off-axis angles can be determined to be

$$
\varphi=\left\{\begin{array}{c}
\arccos \left[\frac{1}{\cos \left(\frac{\theta}{2}\right) \cos \psi}\right. \\
\left.\times\left(\frac{1}{4 m^{2}}\left[1+4 m^{2}+\left(1+8 m^{2}\right)^{1 / 2}\right]-1\right)\right] \\
\text { for } m>1 \\
\arccos \left(\frac{1}{\cos \left(\frac{\theta}{2}\right) \cos \psi}\left(2 m^{2}-1\right)\right) \\
\text { for } m<1 .
\end{array}\right.
$$

If we assume that the transmitting and receiving elevation angles $\theta$ and $\psi$ are very small (which is usually true under practical conditions), which results in $\cos (\theta / 2) \approx 1$ and $\psi \approx 1$, then equation (A11) can be rewritten as

$$
\varphi=\left\{\begin{array}{c}
\arccos \left[\left(\frac{1}{4 m^{2}}\left[1+4 m^{2}+\left(1+8 m^{2}\right)^{1 / 2}\right]-1\right)\right] \\
\text { for } m>1 \\
\arccos \left(2 m^{2}-1\right) \\
\text { for } m<1 .
\end{array}\right.
$$

\section{References}

Aizu Y, Durst F, Gréhan G, Onofri F and Xu T-H 1993 PDA systems without Gaussian beam defects $3 r$ Int. Conf. on Optical Particle Sizing, Yokohama, August 23-26, 1993

Bachalo W D and Sankar S V 1988 Analysis of the light scattering interferometry for spheres larger than the light wavelength 4th Int. Symp. on Applications of Laser Anemometry to Fluid Mechanics, Lisbon, July 11-14, 1988

Bauckhage K 1988 The phase-Doppler-difference-method, a new-laser-doppler technique for simultaneous size and velocity measurements, part 1: description of the method Part. Part. Syst. Charact. 5 16-22

Durst F, Tropea C and Xu T-H 1994 The slit effect in phase Doppler anemometry Proc. 2nd Int. Conf. on Fluid Dynamic Measurement and its Applications ed X Shen and X Sun (Beijing: International Academic Publishers) pp 38-43

Grehan G, Gouesbet G, Naqwi A and Durst F 1991 Evaluation of phase Doppler system using generalized Lorenz-Mie theory Int. Conf. on Multiphase Flows 91, Tsukuba, September 24-27, 1991

Qiu H-H and Hsu C T 1996 A Fourier optics method for the simulation of measurement-volume-effect by the slit constraint 8th Int. Symp. on Applications of Laser Techniques to Fluid Mechanics, Lisbon, July 8-11, 1996 1998a Optimization of optical parameters for particle sizing in multiphase flows by using EPDA Opt. Lasers Eng. 30 3-15 1998b Minimum deviation of spatial frequency in large particle sizing Appl. Opt. 37 6787-94 
Qiu H-H and Sommerfeld M 1992 The impact of signal processing on phase-Doppler measurements 6th Workshop on Two Phase Flow Predictions, Erlangen, March 30-April 2, 1992

Qiu H H, Sommerfeld M and Durst F 1994 Two novel Doppler signal detection methods for laser-Doppler and phase-Doppler anemometry Meas. Sci. Technol. 5 769-78

Saffman M 1986 The use of polarized light for optical particle sizing 3rd Int. Symp. on Applications of Laser Anemometry to Fluid Mechanics, Lisbon, July 14-17, 1986
Sankar S V, Inenaga A S and Bachalo W D 1992 Trajectory dependent scattering in phase Doppler interferometer: minimizing and eliminating sizing errors 6th Int. Symp. on Applications of Laser Anemometry to Fluid Mechanics, Lisbon, July 20-23, 1992

Tropea C, Xu T-H, Onofri F, Gréhan G and Haugen P 1994 Dual mode phase Doppler Anemometry 7th Int. Symp. on Applications of Laser Techniques to Fluid Mechanics, Lisbon, July 8-11, 1994 